\title{
Conditional risk-neutral density from option prices by local polynomial kernel smoothing with no-arbitrage constraints
}

Ana M. Monteiro Antonio A. F. Santos

Monetary and Financial Research Group (GEMF), Centre for Business and Economics Research (CeBER), Faculty of Economics, University of Coimbra

This is a post-peer-review, pre-copyedit version of an article published in Review of Derivatives Research. The final authenticated version is available online at:

https://doi.org/10.1007/s11147-019-09156-x 


\title{
Conditional risk-neutral density from option prices by local polynomial kernel smoothing with no-arbitrage constraints
}

Received: date / Accepted: date

\begin{abstract}
A new approach is considered to estimate risk-neutral densities (RND) within a kernel regression framework, through local cubic polynomial estimation using intraday data. There is a new strategy for the definition of a criterion function used in nonparametric regression that includes calls, puts, and weights in the optimization problem associated with parameters estimation. No-arbitrage constraints are incorporated into the problem through equality and bound constraints. The approach considered yields directly density functions of interest with minimum requirements needed. Within a simulation framework, it is demonstrated the robustness of proposed procedures. Additionally, RNDs are estimated through option prices associated with two indices, S\&P500 and VIX.
\end{abstract}

Keywords Kernel functions · Local polynomials $\cdot$ No-arbitrage constraints · Option prices $\cdot$ Risk-neutral density

\section{Introduction}

Risk-neutral densities (RND) are determinant when dealing with risk management and pricing of new derivative products. In recent years, the amount of information coming from intraday data allows improving and developing existing and new approaches. Nowadays, options are intensively traded in several markets, and these transactions can reveal market expectations on the underlying asset, which are reflected in the corresponding RND. Observed option prices have been used to extract information about the behavior of the underlying asset, since they give insights about risk factors associated with it. RNDs can be used for different purposes, namely to infer about risk in the market, and to price complex option contracts. A central feature that must be considered is that RND estimation is an indirect method as no risk-neutral prices can be observed. 
We propose a novel approach for risk-neutral density estimation within the framework of a nonparametric regression setting (Aït-Sahalia and Lo, 1998; Ait-Sahalia and Duarte, 2003; Yatchew, 2003; Monteiro et al., 2008). The proposed approach can address some main problems presented in previous attempts found in the literature, such as non-monotonicity and non-convexity of call and put pricing estimated functions. There are also problems related to the violation of basic requirements associated with a density or distribution function, its limits, and the non-negativity associated with the density. Additionally, we would expect some degree of smoothness for density estimation since a lack of regularity is not intuitive.

The novel approach is based on a local cubic polynomial kernel regression applied to intraday data. First, it yields the density of interest directly without the need for further transformations. Usually, the literature presents estimation processes that retrieve the call pricing function or its first derivative, and it is necessary to differentiate to obtain the density (Aït-Sahalia and Lo, 1998; Song and Xiu, 2016). Second, it uses compatible information contained in observed call and put intraday prices without using put-call parity. When using intraday data, the difficulty in synchronizing call and put option prices with the underlying asset price can lead to errors (AitSahalia and Lo, 1998; Fengler and Hin, 2015), our approach prevent these to occur. It allows incorporating both prices in the optimization problem, increasing the amount of information that is retrieved from the market, and avoiding errors from lack of synchronicity. Third, it includes smoothly and intuitively no-arbitrage constraints in the optimization problem. Smoothness is mainly a result of incorporating no-arbitrage constraints, and two distinct bandwidths for calls and puts. It allows adapting the optimization problem to different data sets. Also, it takes into account that the first derivative of call and put pricing functions differ from a constant and the second derivatives are equal. Fourth, to account for the relevance of each option price, we introduce open-interest data as weights in the criterion function associated with the problem. By this way, we add more information on market perspectives and beliefs.

The approach is tested against simulated data, which can confirm that the method can recover density functions accurately. When applied to real data sets, constituted by intraday data with less standard behavior, also gives robust and easily interpretable results.

The remainder of the paper is organized as follows, Section 2 discusses the technical details of the risk-neutral density theory. Section 3 introduces the nonparametric estimation based on kernel approaches. Monte Carlo simulated experiments and their discussion are presented in Section 4. Section 5 presents the estimations from market data. In the final section concluding remarks are exposed.

\section{Risk-neutral density through option prices}

Cox and Ross (1976) presented the risk-neutral density in the context of no-arbitrage based models, assuming that investors are risk-neutral. Breeden and Litzenberger (1978) and Banz and Miller (1978) proposed a way of estimating these densities from prices of financial options by considering second derivatives of option pricing functions. 
There are several approaches used to estimate RNDs from option prices. They are extensive and can be divided into two main groups: structural and non-structural models. The former specifies a process for the underlying asset price, and sometimes for volatility. Non-structural models describe the density behavior without prescribing a stochastic process for the underlying asset price. In this last scenario, nonparametric methods and estimation procedures have been developed. They are more flexible and allow for a wide set of shapes. However, for densities in general, larger sample sizes are required. By relaxing assumptions on the underlying process, these models try to achieve a function that describes the data. Utilization of nonparametric estimation methods based on kernel approaches, to obtain a RND implicit in option prices, dates back to the seminal paper of Aït-Sahalia and Lo (1998), revisited by Ait-Sahalia and Lo (2000), Aït-Sahalia et al. (2001), and Aït-Sahalia and Duarte (2003). The main idea was to overcome some drawbacks associated with a parametric setting.

Usually, this kind of nonparametric estimators present rates of convergence substantially lower than their parametric counterparts, and to obtain similar degrees of accuracy far larger sample sizes are needed. This fact is even more relevant when we are considering estimators for first or second derivatives, which is fully addressed by general references for nonparametric methods as Fan and Gijbels (1996), Yatchew (2003), Härdle (1990), Li and Racine (2007), and for the specific application to RND estimation by Aït-Sahalia and Duarte (2003).

There are different goals when considering kernel estimation procedures dealing with no-arbitrage constraints. Jackwerth (2000) computed a subjective distribution based on a kernel estimator. Rosenberg and Engle (2002) estimated risk aversion by considering a kernel function depending on the maturity. Aït-Sahalia and Duarte (2003) proposed shape restrictions for the optimization problem considering local polynomial estimation. Yatchew and Härdle (2006) and Härdle and Hlávka (2009), using smoothing splines, also imposed constraints on the estimation problem, in order to guarantee convexity and monotonicity for call pricing functions. Monteiro et al. (2008) proposed a nonparametric approach with no-arbitrage constraints based on cubic splines through a semidefinite programming problem. Zhang et al. (2009) considered a local polynomial estimator together with a method based on Gram-Charlier series expansion to obtain RNDs. Song and Xiu (2016) considered a nonparametric kernel approach for estimate RND, including volatility factors. They used local polynomial linear estimators for first derivatives of option pricing functions, using end-of-day data from S\&P500 and VIX.

Data length is an essential issue in any estimation process. Ait-Sahalia and Lo (1998), and most of the subsequent studies, considered extensive time series. Most papers from the literature consider data from a significant period and assume the estimated risk-neutral density as an average of densities. Recently, intraday data has become more accessible, and the amount of data collected in a few days gives enough information to infer RNDs. Dalderop (2018) estimates time-varying RNDs by considering a kernel estimator as a function of time and moneyness, applied to intraday data. The author uses different order approximations: local constant for time dimension and local cubic for moneyness.

Several frameworks have been used to derive call option pricing functions, which by no-arbitrage arguments must be associated with a portfolio without risk and a 
risk-free interest rate. It has been found that corresponds to calculate the expected future option value at expiration, computed through a risk-neutral density measure, $\mathbb{Q}$, discounted by the risk-free interest rate, which can be expressed as

$$
\begin{aligned}
C\left(S_{t}, X, \sigma_{t}, \tau, r, \boldsymbol{\delta}\right) & =e^{-r \tau} E^{\mathbb{Q}}\left[\left(S_{T}-X\right)^{+}\right] \\
& =S_{t} \mathbb{Q}^{S}\left(S_{T}>X\right)-X e^{-r \tau} \mathbb{Q}\left(S_{T}>X\right) \\
& =S_{t} P_{1}-X e^{-r \tau} P_{2} .
\end{aligned}
$$

where $t$ represents the current date, $X$ the strike price, $r$ the risk-free interest rate, $S_{t}$ the current underlying asset price, $S_{T}$ the asset price at maturity, $\tau=T-t$ the time-to-maturity, and $\delta$ the dividend yield. The main difference from Black-Scholes' formula is related to probabilities $P_{1}$ and $P_{2}$, measured through risk-neutral densities. The formula reveals that option price depends mainly on the underlying asset and strike prices. Different variations can be considered by changing the structure of $P_{1}$ and $P_{2}$, which allows the use of mean pricing functions in different contexts through minor changes.

Consider an economy with two state variables, the price of S\&P 500 index $S$, and an unobserved volatility $V$. Since the volatility is determinant for pricing $S$, also consider the VIX option market and denote the variable by $Z$. As there are no contingent claims written on $V$, we will consider option contracts on the volatility index VIX in order to estimate the risk-neutral densities of $S$ and $Z$.

The European call option price for contracts on $\mathrm{S}$ can be given by

$$
C\left(S_{t}, X, \sigma_{t}, \tau, r, \delta\right)=e^{-r \tau} \int_{X}^{\infty}\left(S_{T}-X\right) g\left(S_{T} \mid S_{t}, \sigma_{t}, r, \delta, \tau\right) d S_{T},
$$

where $g(\cdot)$ represents the conditional risk-neutral density for the underlying asset at expiration T. Considering the same assumptions, the price of a European put option is

$$
P\left(S_{t}, X, \sigma_{t}, \tau, r, \delta\right)=e^{-r \tau} \int_{0}^{X}\left(X-S_{T}\right) g\left(S_{T} \mid S_{t}, \sigma_{t}, r, \delta, \tau\right) d S_{T} .
$$
by

The price of a European call option on VIX, with strike $L$, can be given similarly

$$
J\left(Z_{t}, L, \sigma_{t}, \tau, r, \delta\right)=e^{-r \tau} \int_{X}^{\infty}\left(Z_{T}-L\right) h\left(Z_{T} \mid Z_{t}, \sigma_{t}, r, \delta, \tau\right) d Z_{T},
$$

where $Z_{T}$ is the VIX index price on the maturity, and $h(\cdot)$ is the conditional riskneutral density for $Z_{T}$.

Breeden and Litzenberger (1978) and Banz and Miller (1978) proposed a relationship between second derivative of the call option price, with respect to the strike price, and the risk-neutral density:

$$
\begin{aligned}
\frac{\partial C\left(S_{t}, X, \sigma_{t}, \tau, r, \delta\right)}{\partial X} & =\frac{\partial\left(e^{-r \tau} \int_{X}^{\infty}\left(X-S_{T}\right) g\left(S_{T} \mid S_{t}, \sigma_{t}, r, \delta, \tau\right) d S_{T}\right)}{\partial X} \\
& =e^{-r \tau}\left(G\left(X \mid S_{t}, \sigma_{t}, r, \delta, \tau\right)-1\right),
\end{aligned}
$$


where $G(\cdot)$ is the respective cumulative distribution function associated with $g(\cdot)$. The second derivative is then expressed as

$$
\frac{\partial^{2} C\left(S_{t}, X, \sigma_{t}, \tau, r, \delta\right)}{\partial X^{2}}=e^{-r \tau} g\left(X \mid S_{t}, \sigma_{t}, r, \delta, \tau\right)
$$

and the risk-neutral density at expiration is

$$
g\left(X \mid S_{t}, \sigma_{t}, r, \delta, \tau\right)=\left.e^{r \tau} \frac{\partial^{2} C\left(S_{t}, X, \sigma_{t}, \tau, r, \delta\right)}{\partial X^{2}}\right|_{X=S_{T}} .
$$

The risk-neutral density can be established in an equivalent form using puts as was considered for calls,

$$
\frac{\partial P\left(S_{t}, X, \sigma_{t}, \tau, r, \delta\right)}{\partial X}=e^{-r \tau} G\left(X \mid S_{t}, \sigma_{t}, r, \delta, \tau\right)
$$

and

$$
\begin{gathered}
\frac{\partial^{2} P\left(S_{t}, X, \sigma_{t}, \tau, r, \delta\right)}{\partial X^{2}}=e^{-r \tau} g\left(X \mid S_{t}, \sigma_{t}, r, \delta, \tau\right) . \\
g\left(X \mid S_{t}, \sigma_{t}, r, \delta, \tau\right)=\left.e^{r \tau} \frac{\partial^{2} P\left(S_{t}, X, \sigma_{t}, \tau, r, \delta\right)}{\partial X^{2}}\right|_{X=S_{T}} .
\end{gathered}
$$

Considering VIX options

$$
\begin{aligned}
\frac{\partial J\left(Z_{t}, L, \sigma_{t}, \tau, r, \delta\right)}{\partial L} & =\frac{\partial\left(e^{-r \tau} \int_{X}^{\infty}\left(L-Z_{T}\right) h\left(Z_{T} \mid Z_{t}, \sigma_{t}, r, \delta, \tau\right) d Z_{T}\right)}{\partial L} \\
& =e^{-r \tau}\left(H\left(L \mid Z_{t}, \sigma_{t}, r, \delta, \tau\right)-1\right)
\end{aligned}
$$

where $L$ represents the strike price, and $H(\cdot)$ is the respective cumulative distribution function associated with $h(\cdot)$

$$
h\left(Z_{T} \mid Z_{t}, \sigma_{t}, r, \delta, \tau\right)=\left.e^{r \tau} \frac{\partial^{2} J\left(Z_{t}, L, \sigma_{t}, \tau, r, \delta\right)}{\partial L^{2}}\right|_{L=Z_{T}} .
$$

By combining first and second derivatives of call and put pricing functions, we obtain constraints to be imposed on our optimization problem. Since pricing functions, in this context, are homogeneous of degree one in the strike (Fengler and Hin, 2015; Song and Xiu, 2016), we can scale strikes and prices without changing the relationship between variables.

\section{Nonparametric estimation}

A cornerstone for nonparametric methods applied to RND estimation is the seminal paper of Ait-Sahalia and Lo (1998). It is stressed out its importance, and how with sufficient amount of data it is possible to get rid of ties associated with parametric approaches.

Nonparametric approaches offer more flexible methods for modeling the evolution of mean option pricing function on strikes, and other relevant variables. The 
second derivative is related to RNDs. Using Aït-Sahalia and Lo (1998) notation, suppose there is a smooth function $H(\cdot)$ that can be seen as an option pricing function depending on a vector $U$, set as $U=\left(S_{t}, X, \tau, r, \delta\right)$. A possible nonlinear relationship is established as

$$
H_{i}=H\left(U_{i}\right)+\varepsilon_{i}, \quad i=1, \ldots, n .
$$

In this formulation, $\varepsilon_{i}$ is an error term expressing the fact that observed option prices include measurement errors, possibly heteroscedastic, meaning that the error terms are not identically distributed.

Nadaraya-Watson (NW) estimator is commonly used (Nadaraya, 1964; Watson, 1964), and assumes the form

$$
\hat{H}\left(U_{i}\right)=\frac{\sum_{i=1}^{n} K_{h}\left(U_{i}-U\right) H_{i}}{\sum_{i=1}^{n} K_{h}\left(U_{i}-U\right)},
$$

which can be seen as a weighted mean average of the $U_{i}^{\prime} s$. A kernel function $K_{h}(\cdot)$, depending on a bandwidth $h$, for a given point $U$, defines the weights. The application of this estimator can be challenging. As nonparametric methods are data intensive, their effectiveness rapidly decreases as the problem's dimension increases (number of explanatory variables). Without imposing adequate constraints, estimates can contradict basic economic principles and even common sense. To overcome this problem Aït-Sahalia and Lo (1998) proposed some justifications for dimension reduction. They estimated RNDs by an indirect way, through an estimator for implied volatility, which is plugged-in Black-Scholes' formula. By this approach, it is natural that RNDs inherit most characteristics obtained using a parametric model as the one referred.

To address some problems associated with estimation procedures proposed in Ait-Sahalia and Lo (1998), Ait-Sahalia and Duarte (2003) revisited the problem, and a new approach was proposed that has been followed in subsequent literature. Instead of local constant polynomial kernel regression, a more general setting was proposed based on local linear polynomial regression, but more importantly, it was highlighted the importance of shape restrictions on mean pricing functions, and respective derivatives, for obtaining meaningful results. A univariate setting is adopted making option prices depending only on strikes, and obtaining similar accuracy using fewer observations. Estimators were subjected to a series of shape constraints, and a kind of double smoothing. They devised a two-step procedure which incorporates a complex optimization problem, followed by a kernel smoothing estimation for obtaining the desired densities.

NW estimator can be seen as a local constant polynomial kernel regression type estimator. A more general approximation can soften some drawbacks associated with it. Let us consider for simplicity a general formulation $y_{i}=m\left(x_{i}\right)+\varepsilon_{i}$. For a local polynomial of order $p$, kernel regression estimators are obtained by solving the problem

$$
\text { minimize } \sum_{i=1}^{n}\left(y_{i}-\sum_{k=0}^{p} \beta_{k}(x) \frac{\left(x_{i}-x\right)^{k}}{k !}\right)^{2} K\left(\frac{x_{i}-x}{h}\right),
$$

where the decision vector is $\beta(x)$. This general formulation encompasses constant $(p=0)$, linear $(p=1)$, quadratic $(p=2)$, and cubic $(p=3)$ orders. Compared with 
NW estimator, nonparametric local linear polynomial approximation $(p=1)$ represents an important improvement in terms of flexibility and estimator's properties (Fan and Gijbels, 1996). Applying a local $p$-order polynomial criterion, the mean function estimator, and respective derivatives, are given directly by $\hat{m}(x)=\hat{\beta}_{0}(x)$, and $\hat{m}^{(k)}(x)=k ! \hat{\beta}_{k}(x)$.

Several authors accommodate no-arbitrage constraints in the definition of nonparametric estimates for RNDs, as already mentioned. Aït-Sahalia and Duarte (2003), but also Yatchew and Härdle (2006), used constrained nonparametric least squares problem, where constraints are defined through a penalty component. Yatchew and Härdle (2006) consider Sobolev norms to calculate a matrix called representor, making this approach less intuitive, and not easy to implement. Birke and Pilz (2008) address the problem using an auxiliary inverse function associated with call pricing function first derivative, that needs to be integrated or differentiated for obtaining call pricing functions or risk-neutral densities, respectively.

We devise an alternative procedure in comparison with those mentioned above. In contrast, a simple and intuitive framework to include no-arbitrage constraints directly in a criterion function is developed. A fact not fully explored in literature is related to the derivation of risk-neutral densities using information contained directly in both calls and puts, without using put-call parity. As functions of strikes, call and put pricing functions move in opposite directions, and also the variability. In left tail, call prices vary more than puts, and vice versa in the right tail. Expressing parameters, in a criterion function, as values of risk-neutral distributions and densities (the latter coincides for calls and puts) allows easily to impose no-arbitrage constraints. The contrast (variability) between call and put prices can be used as a valuable source of information to define robust estimation procedures.

\subsection{Nonparametric with no-arbitrage constraints}

When RNDs are estimated implicitly through option prices, it is desired to obtain a smooth function. The most area must be associated with a neighborhood around the current value of the underlying asset. On tails direction, density values must tend to zero. A fundamental problem is how tails behave, their rate of convergence to zero, and comparisons between left and right tails.

No-arbitrage constraints are intimately related to monotonicity and convexity that are established characteristics of call and put pricing functions. Following Birke and Pilz (2008), no-arbitrage constraints assume the form

$$
\begin{aligned}
-e^{-r \tau} \leq \frac{\partial C}{\partial X}(X) & \leq 0 \\
\frac{\partial^{2} C}{\partial X^{2}}(X) & \geq 0 \\
C(X) & \geq 0, \quad \forall X \in[0, \infty[
\end{aligned}
$$


By using put-call parity, the same kind of constraints can be associated with put pricing functions,

$$
\begin{aligned}
0 \leq \frac{\partial P}{\partial X}(X) & \leq e^{-r \tau} \\
\frac{\partial^{2} P}{\partial X^{2}}(X) & \geq 0 \\
P(X) & \geq 0 \quad \forall X \in[0, \infty[.
\end{aligned}
$$

Using these constraints, we define an extended criterion function within a constraint nonparametric regression framework.

Let us designate $c_{i}$ and $p_{j}$ observed prices for calls and puts, with respective strikes $x_{i}$ and $x_{j}$, for $i=1, \ldots, n$ and $j=1, \ldots, m$. The proposed criterion function is an extension of a local cubic polynomial approximation within a nonparametric regression setting. The extension accounts jointly call and put prices. This approach has the advantage of representing a contrast of information, and also allows no-arbitrage constraints straightforwardly.

In a kernel regression framework, where local approximations are defined around $x$, kernel functions serve to weight the distance of sample observations to $x$. Two kernel functions are considered, $K\left(\left(x_{i}-x\right) / h_{c}\right)$ for calls, and $K\left(\left(x_{j}-x\right) / h_{p}\right)$ for puts, accounting for different bandwidths, $h_{c}$ and $h_{p}$. As it is well documented in the literature (Härdle, 1990; Fan and Gijbels, 1996; Yatchew, 2003; Li and Racine, 2007), these parameters have a major influence, namely, in comparison with the choice of the kernel function. This fact leads to consider only Gaussian kernels, although different kernels were tested without significant changes.

Within the regression framework considered, it is apparent that error's terms variance is not constant, a fact also emphasized in Yatchew and Härdle (2006) in the context of RND estimation. To cope with this data characteristic, different weights could be attributed to different observations. More informative ones are represented by at-the-money prices, deep-in-the-money or deep-out-of-the-money are less informative. These can be weighted by volume or open-interest values, represented for calls by $w_{i, c}$, and for puts by $w_{j, p}$.

The estimation is performed by minimizing a criterion function subject to the set of linear and bound constraints 1-10,

$$
\begin{aligned}
\operatorname{minimize} & \sum_{i=1}^{n} w_{i, c}\left(c_{i}-\beta_{0, c}(x)-\sum_{k=1}^{3} \beta_{k, c}(x) \frac{\left(x_{i}-x\right)^{k}}{k !}\right)^{2} K\left(\frac{x_{i}-x}{h_{c}}\right)+ \\
& \sum_{j=1}^{m} w_{j, p}\left(p_{j}-\beta_{0, p}(x)-\sum_{k=1}^{3} \beta_{k, p}(x) \frac{\left(x_{j}-x\right)^{k}}{k !}\right)^{2} K\left(\frac{x_{j}-x}{h_{p}}\right)
\end{aligned}
$$


subject to

$$
\begin{aligned}
-\beta_{1, c}(x)+\beta_{1, p}(x) & =e^{-r \tau} \\
\beta_{2, c}(x)-\beta_{2, p}(x) & =0 \\
\max \left(0, S_{t}-x e^{-r \tau}\right) \leq \beta_{0, c}(x) & \leq S_{t} \\
\max \left(0, x e^{-r \tau}-S_{t}\right) \leq \beta_{0, p}(x) & \leq \infty \\
-e^{-r \tau} \leq \beta_{1, c}(x) & \leq 0 \\
0 \leq \beta_{1, p}(x) & \leq e^{-r \tau} \\
\beta_{2, c}(x) & \geq 0 \\
\beta_{2, p}(x) & \geq 0 .
\end{aligned}
$$

For each local approximation at $x$, the problem (11-19) can be characterized as a Generalized Least Squares (GLS) problem with constraints, which can be solved as a Quadratic Programming (QP) problem. Let us designate $y$ as the observations vector for call and put prices, and consider matrices $X_{c}(x)$ and $X_{p}(x)$, with typical rows $i$ and $j$ given by

$$
\begin{aligned}
X_{i, c}(x) & =\left[\begin{array}{ll}
1\left(x_{i}-x\right) & (1 / 2)\left(x_{i}-x\right)^{2}(1 / 6)\left(x_{i}-x\right)^{3}
\end{array}\right] \\
X_{j, p}(x) & =\left[\begin{array}{ll}
1\left(x_{j}-x\right) & (1 / 2)\left(x_{j}-x\right)^{2}(1 / 6)\left(x_{j}-x\right)^{3}
\end{array}\right]
\end{aligned}
$$

for the vectors and matrix defined as

$$
y=\left[\begin{array}{l}
c \\
p
\end{array}\right], \beta=\left[\begin{array}{l}
\beta_{c} \\
\beta_{p}
\end{array}\right], X=\left[\begin{array}{cc}
X_{c}(x) & \mathbf{0} \\
\mathbf{0} & X_{p}(x)
\end{array}\right]
$$

Given a matrix of weights represented by $W$, and a "kernel matrix" $K=K\left(h_{c}, h_{p}\right)$, the minimization problem (11-19) is expressed as a quadratic optimization problem

$$
\begin{aligned}
& \operatorname{minimize}(y-X \beta)^{\top} W^{1 / 2} K W^{1 / 2}(y-X \beta) \\
& \text { subject to } \quad \beta \in \mathscr{B}
\end{aligned}
$$

where $\mathscr{B}$ is the set of constraints. By considering

$$
\begin{aligned}
y^{*} & =W^{1 / 2} K^{1 / 2} y \\
X^{*} & =W^{1 / 2} K^{1 / 2} X
\end{aligned}
$$

the latter can be rewritten as a norm minimization problem subject to convex constraints,

$$
\begin{aligned}
& \operatorname{minimize}\left\|y^{*}-X^{*} \beta\right\| \\
& \text { subject to } \quad \beta \in \mathscr{B}
\end{aligned}
$$

which can be translated to a QP optimization problem

$$
\begin{aligned}
& \text { minimize } \beta^{\top} H \beta+f^{\top} \beta \\
& \text { subject to } \quad \beta \in \mathscr{B}
\end{aligned}
$$

where $H=X^{* \top} X^{*}$ and $f=-X^{* \top} y^{*}$. 
By applying a local cubic polynomial approximation, at each point $x$ we obtain estimates for $\beta_{i, j}(x)$, with $i=0,1,2,3, j=c, p$. The main aim are the estimates $\hat{\beta}_{2, c}(x)$ and $\hat{\beta}_{2, p}(x)$, with $\hat{\beta}_{2, c}(x)=\hat{\beta}_{2, p}(x)$, that represent the risk-neutral-density's value at $x$. Equally important are constraints implied by no-arbitrage arguments, that act as smoothing components to obtain more reliable and intuitive RND estimates.

\subsection{Bandwidths and weights selection}

In nonparametric regression methods frameworks, kernel functions measure, locally, the distance to a point $x$. Kernel functions are symmetric around $x$, and integrate one. Its value depends on the distance of observations to $x$, scaled by a bandwidth parameter, which is recognized as the most relevant factor in terms of characteristics and quality of model fitting.

When approximating a mean function, using the Mean-Square Error (MSE) criterion, an optimal bandwidth is chosen through a min-max optimization problem, which is related to a trade-off between bias and variance. An MSE criterion allows the definition of a local optimal bandwidth, which depends on many factors, for example, sample size, curvature of the mean function, distribution of design variables, and their respective variance. Usually, these quantities are unknown.

Local optimal bandwidths are challenging to define. A common approach tries to approximate a global optimal bandwidth, which is defined through the minimization of the Mean Integrated Square Error (MISE). In some cases, it is possible to define an analytic expression to the global bandwidth. However, it depends on unknown quantities. In practical terms, to define the optimal global bandwidth, Cross Validation $(\mathrm{CV})$ methods are used. An obtained value is asymptotically optimal through the MISE criterion.

In our approach, two bandwidths were considered, $h_{c}$ related to call observations, and $h_{p}$ to puts. We apply CV to obtain a first approximation as only observed values and estimated means can be compared. Considering again a general setting, $y_{i}=$ $m\left(x_{i}\right)+\varepsilon_{i}, i=1, \ldots, n$, and using the common approach for $\mathrm{CV}$, which is leave-oneout, $h$ is chosen by

$$
\text { minimize } C V(h)=\frac{1}{n} \sum_{i=1}^{n}\left(y_{i}-\hat{m}_{-i}\left(x_{i}\right)\right)^{2} W\left(x_{i}\right),
$$

where $\hat{m}_{-i}\left(x_{i}\right)$ is the leave-one-out kernel estimator of $m\left(x_{i}\right)$, and $W(\cdot)$ is a weight function, see Li and Racine (2007).

Adapting CV to define $h_{c}$ and $h_{p}$, we take into account a fixed design framework, where for each $x_{i}, i=1, \ldots, n, k_{i}$ observations for $y$ are available, which means that we have to implement the procedure leave- $k_{i}$-out. The CV criterion is modified, and bandwidths are chosen by

$$
\begin{aligned}
\operatorname{minimize} C V\left(h_{c}, h_{p}\right)= & \frac{1}{n} \sum_{i=1}^{n} \sum_{j=1}^{k_{i}}\left(y_{c, j}-\hat{m}_{-k_{i}}^{c}\left(x_{i}\right)\right)^{2} W_{c}\left(x_{i}\right)+ \\
& \frac{1}{n} \sum_{i=1}^{n} \sum_{j=1}^{k_{i}}\left(y_{p, j}-\hat{m}_{-k_{i}}^{p}\left(x_{i}\right)\right)^{2} W_{p}\left(x_{i}\right),
\end{aligned}
$$


where kernel estimator for calls, $\hat{m}_{-k_{i}}^{c}\left(x_{i}\right)$, depend on $h_{c}$ and for puts, $\hat{m}_{-k_{i}}^{p}\left(x_{i}\right)$, depend on $h_{p}$. Weighing functions, $W_{c}\left(x_{i}\right)$ and $W_{p}\left(x_{i}\right)$, will also be different for calls and puts, they reflect choices done when the criterion function, for parameters estimation, was defined.

Weights used for parameters estimation and $\mathrm{CV}$, account for the distance between observations and a point representing at-the-money prices. As $h_{c}$ and $h_{p}$ define a neighborhood around $x$, elements of $W_{c}(\cdot)$ and $W_{p}(\cdot)$ represent distance to a point where observations carry more information (at-the-money). In this paper, the proxies considered for these weighting functions are open-interest values, associated with call and put option contracts.

\section{Monte Carlo analysis}

In this section, we present a simulation analysis that demonstrates the effectiveness of the methods proposed in this study. Risk-neutral prices are not directly observable but can be inferred indirectly through option prices. Except in the case of simulated data, no confrontation between true and estimated RND can be done.

It is assumed that the stochastic process associated with an underlying asset is given by a diffusion process subjected to stochastic volatility, which can be represented as

$$
\begin{aligned}
d S_{t} & =\mu d t+\sqrt{v_{t}} S_{t} d W_{t} \\
d v_{t} & =\kappa\left(\theta-v_{t}\right) d t+\sigma \sqrt{v_{t}} d Z_{t},
\end{aligned}
$$

where $W_{t}$ and $Z_{t}$ are two standard Brownian motion processes with $E\left(d W_{t} d Z_{t}\right)=$ $\rho d t, \kappa$ represents the mean-reverting volatility parameter, $\theta$ the long-run volatility, and $\sigma$ the volatility of volatility. Under certain assumptions, given in Heston (1993), there is a closed form solution for European-type option prices. The assumptions are related to a risk-premium function and the current value of volatility. Henceforth, assuming a given value for the current volatility, prices of calls and puts for different strike values are generated by what we refer as Heston (1993) model.

The parameters adopted are $\kappa=5, \theta=0.03, \sigma=0.3$, and $\rho=-0.7$. In the simulation a zero dividend yield, a risk-free rate $r=0.02$, and a time to maturity of 3 -months $(\tau=0.25)$, are adopted. It is assumed that at $t$ the price of the underlying asset is $S_{t}=50$, and the range of strikes is given by the interval [35,62]. For prices obtained using the model, random noise was added to mimic observed market prices. Using these perturbed prices, we illustrate the performance of nonparametric methods developed in this paper. With Heston's model, no analytic formula is available to express RNDs. Nowadays, we can generate Heston's prices for calls and puts within a fine grid of strikes, and approximate RNDs smoothly and accurately using second derivatives calculated numerically. For parameters' values defined above, this kind of calculations was performed and are depicted in Figure 1. An equally-spaced of fine strike prices was considered and numerical second derivatives were calculated, presenting high stability. True RND can be obtained with high-accuracy, this function a positive and integrates one. It presents a slightly negative skewness, corresponding to a negative value assumed by the leverage effect $\rho=-0.7$. 

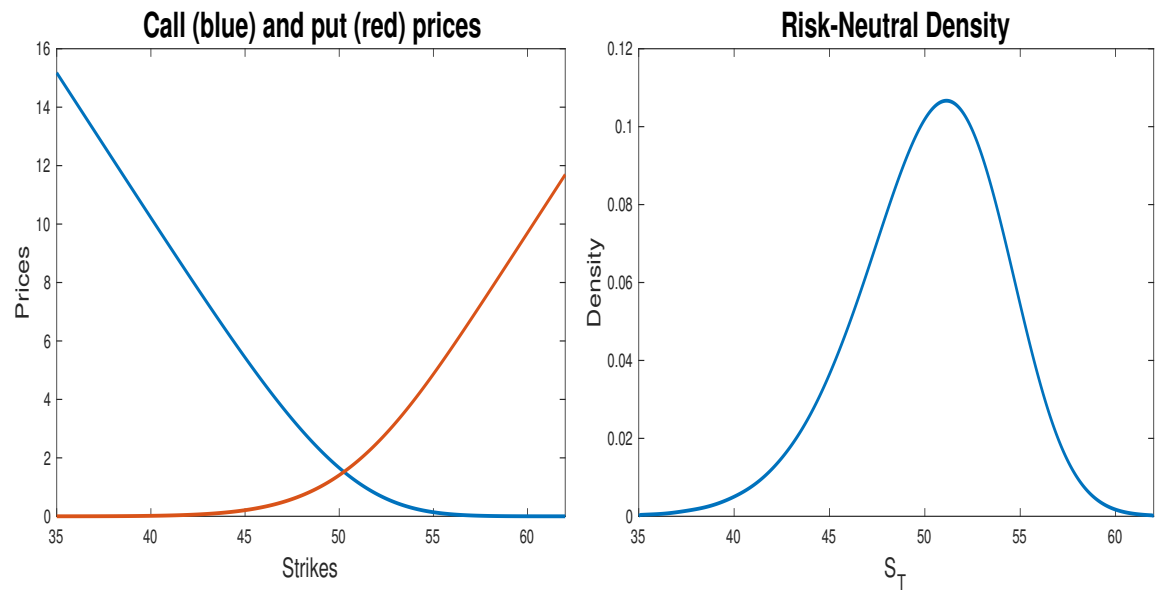

Fig. 1 Heston's model prices and RND.
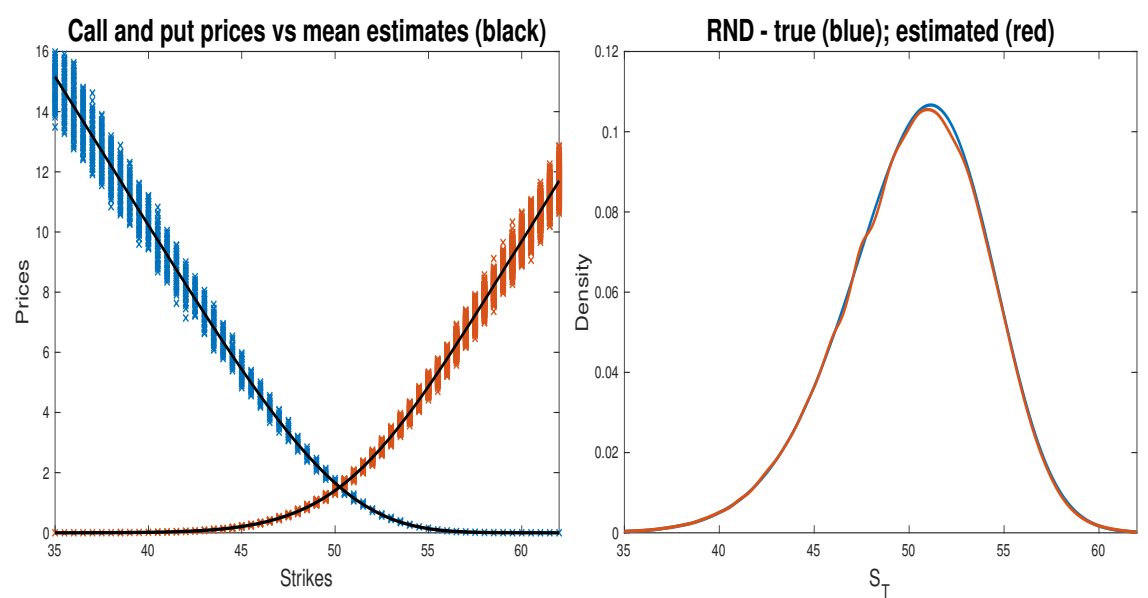

Fig. 2 Simulated prices; mean and RND estimates.

To reproduce market data, for each strike a theoretical price is calculated, and a series of observations are simulated adding some noise to the prices. Following Yatchew and Härdle (2006), and assuming already a nonparametric framework, we used the formula $y_{i}=m\left(x_{i}\right)+0.03 m\left(x_{i}\right) \varepsilon_{i}$, with $\varepsilon_{i} \sim N(0,1)$, where for each strike one thousand observations were simulated. Resulting prices and comparisons between true and estimated RND are depicted in Figure 2. We obtain a good overall fitting and the expected behavior for density tails. In the simulation, we have a very controlled environment with equally-spaced strikes, an equal number of observations for each strike; however, prices are highly perturbed. As it is also demonstrated in the literature associated with RND estimation, obtaining a good fit for the mean pricing function does not constitute a great challenge, in opposition to its second derivative 

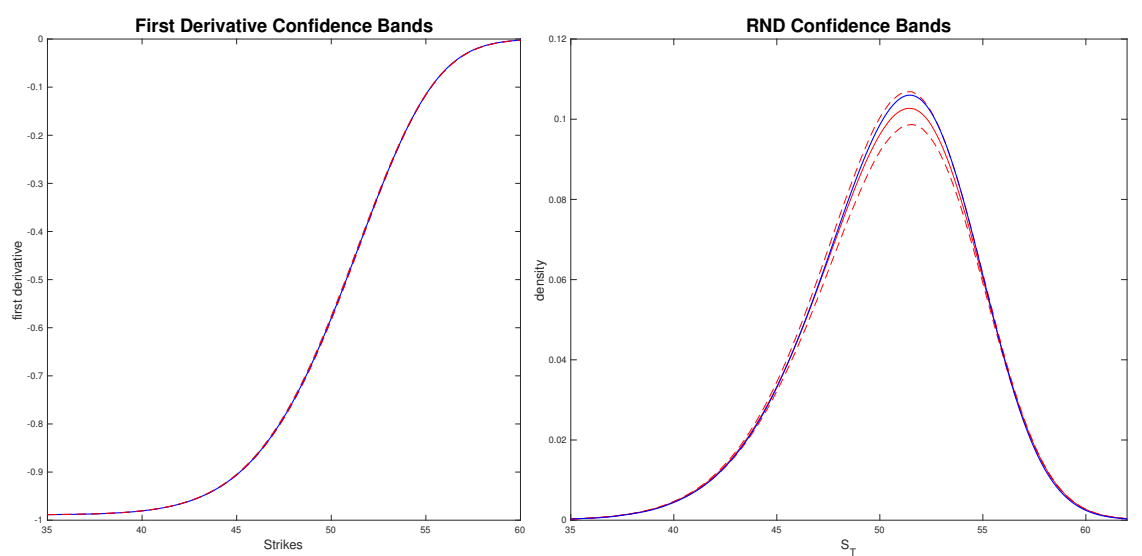

Fig. 3 Simulated 95\% confidence intervals for first derivatives and RND estimates (red dashed lines); true functions (blue solid lines); median (red solid line).

estimation (Aït-Sahalia and Duarte, 2003; Yatchew and Härdle, 2006; Birke and Pilz, 2008; Grith et al., 2012).

Regarding the robustness of methods proposed in this article, we present simulated 95\% confidence intervals for first derivatives and RND estimates. Our results are comparable with the ones in Aït-Sahalia and Duarte (2003) meaning that estimates, and respective confidence intervals, are conformable with option pricing and statistical theory, e.g., no-arbitrage constraints and proper density functions. The simulation setting is slightly different but comparable. We have used 79 observations for each strike price assuming Heston's model, with parameters defined previously. The results are depicted in Figure 3, in blue we have the true RND, in red the median estimates (solid line), and dashed lines refer to $95 \%$ confidence intervals. They are obtained using a Monte Carlo approximation through 5000 iterations, from which the respective quantiles were estimated.

Estimation methods perform satisfactorily, with very narrow confidence intervals, especially for first derivatives, and RND tails. Limits of confidence intervals for first derivatives are so narrow that are barely discernible in the figure. For RND, confidence intervals are well visible, narrow and meaningful in the tails, and as it would be expected wider in center where the curvature is more pronounced. This fact is well-established regarding the properties of general nonparametric estimators, which depend heavily on second derivatives.

\section{Empirical demonstration}

In this section, we provide nonparametric density estimations using S\&P500 and VIX options. Each underlying asset gives rise to a massive number of option contracts, with different maturities available, and also many strikes. Considering only vanilla options, a significant amount of put and call contracts makes difficult data retrieving, 
treatment and application of common data-cleaning procedures. For these tasks, it is necessary to build data structures able to accommodate the diversity and complexity in data. The number of contracts and maturity dates available for a given stock can be different for calls and puts. Finally, most trades are done near-the-money, which varies with the underlying asset price. Option contracts that are deep-in-the-money or deep-out-of-the-money are rarely traded, which means that observed prices can carry different amounts of information.

\subsection{Data description}

We use intraday data for options associated with two indices, S\&P500 and VIX, from CBOE. Options related to S\&P500 index correspond to the SPXW version of contracts. Data was collected from the publicly available site YahooFinance using tailored software to record observations for every contract (strikes, maturities), during regular daily negotiation time. The sample corresponds to observations obtained from April 16 to April 20, 2018.

Despite the short period represented by one week, due to the diversity as mentioned earlier, the number of observed contracts are of orders $10^{4}$ and $10^{3}$. Added to this fact, the frequency of observed data is 5 minutes, and each contract was considered 79 times in the sample. For SPXW with time-to-maturity of around one month, 15964 and 21508 observations were considered for calls and puts, respectively, whereas, for VIX, 1465 and 1196 observations are available. These numbers are comparable with ones considered in literature that uses nonparametric methods to estimate RND functions, see e.g. Aitt-Sahalia and Lo (1998), Song and Xiu (2016).

Following the literature, it can be clearly understood that due to heterogeneity associated with options data, data-cleaning procedures are needed. The main aim is to remove troublesome data points in terms of compatibility with theoretical results, e.g., no-arbitrage constraints, and points that are irrelevant as correspond to contracts that have never been traded. In our approach, data-cleaning is reduced to minimal procedures. First, we eliminate duplicate observations that result from working with high-frequency data. Second, option contracts with bid or open interest equal to zero are also eliminated. This last step assumes less importance since we use open-interest as a weight in the estimation procedures, which eliminates automatically such observations.

In the literature, out-of-the-money and in-the-money call and put prices are considered separately because they accommodate different information. The well-known put-call parity formula is used, converting put into call prices. This conversion is not absent from difficulties, mainly because lack of synchronization, which can only be softened using end-of-day data (Song and Xiu, 2016), but not entirely resolved. We include directly in estimation procedures call and put contract prices. By taking advantage of this inclusion, avoiding using put-call parity conversions, new information is added allowing better estimates to be obtained.

As the sample period considered is short, less variation of elements that determine option prices need to be accounted for. Examples are the risk-free interest rate, dividend yield, and underlying stock prices. Long sample periods may raise concerns 

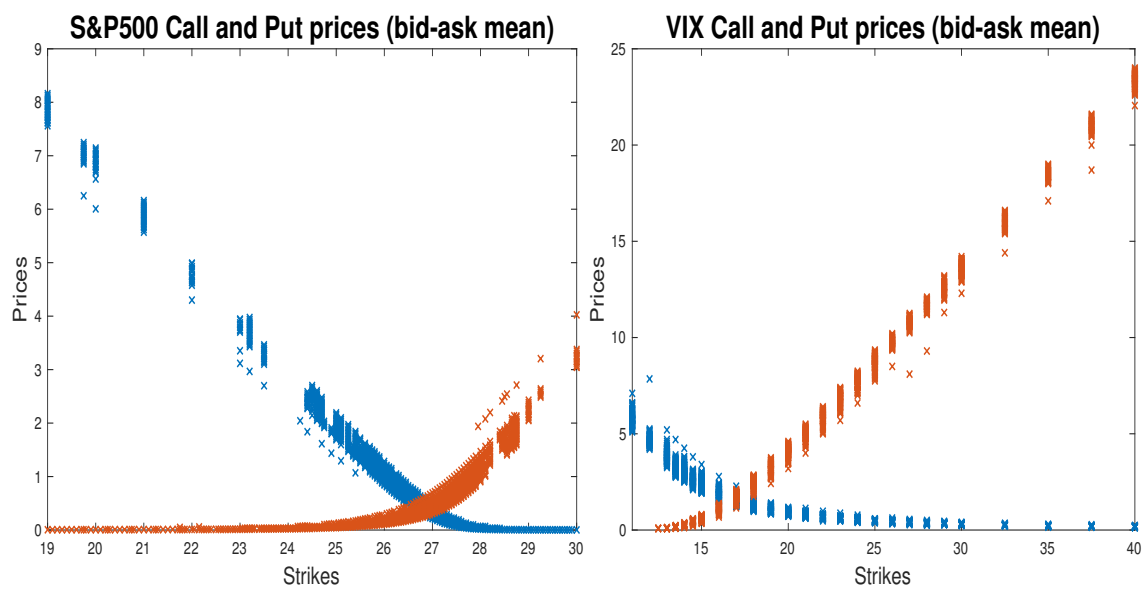

Fig. 4 Call and put bid-ask mean prices with expiration date May 18, $2018(\tau=1 / 12)$ on S\&P 500 index, and May 16, $2018(\tau=1 / 12)$ on VIX index.

about structure maintenance of prices. Contrasting with Aït-Sahalia and Lo (1998) that have considered, around one year of daily data, and Song and Xiu (2016) with seventeen years, we use a short period of intraday data that allows us to compare results with the referred ones in terms of data dimension. As we deal with a short sample period, we only considered contracts with a fixed short maturity date. Timeto-maturity is fixed, and in RND estimation setting, this resembles a cross-section approach.

Aït-Sahalia and Lo (1998) and Song and Xiu (2016) performed an analysis by rolling forward contracts, and defined results for a mean maturity period. Due to using intraday data, we do not need such data manipulation procedure. By considering options in the sample mentioned above, a time-to-maturity of around one month was considered, May 18 for S\&P500, and May 16 for VIX. Bid-ask mean prices were used to represent observed prices, and for each strike, a series was obtained, for calls and puts. Data is depicted in Figure 4, where graphics were truncated to show the most relevant parts. For S\&P500, strikes and prices were scaled by a $10^{-2}$ factor, representing a change of unity justified by pricing functions homogeneity, and allowing better visualization of data.

\subsection{Estimation results}

Using intraday data, a series of prices is obtained for each strike, and a mean function can be approximated by simple averaging option prices. Considering yet a nonparametric approach, even with a local constant estimator (Nadaraya-Watson), mean function estimates cannot be ruled out by any economic or statistical argument. It is assumed that option pricing functions are twice differentiable, and are expected to be monotone and convex, consequently for an interior point the estimator is consistent. However, when first and second derivatives are estimated, statistical properties de- 
grade with a substantial decrease of convergence rates. More importantly, estimates start to lack economic sense, and go against established theoretical results. These facts are well-established in Aït-Sahalia and Duarte (2003) and Yatchew and Härdle (2006), which constitute main motivations for presenting new methods for dealing with such drawbacks.

Considering the estimation applied to S\&P500 data set (Figure 4), as we used one hundred units to refer S\&P500 data, the less troublesome region is defined by strikes between 24 and 28 for calls and puts, respectively. Below strike 24, for calls, strikes grid is sparser, the same happens for puts with values above 28. Regions where information for calls is lacking are compensated by information from puts, and vice versa. For the performed estimation, time-to-maturity was set to $\tau=1 / 12$ and, based on Treasury Bills data, a value of $2 \%$ was considered to be the risk-free interest rate $r$. Our main contribution was to devise a method able to cope with such different sets within the estimation of a unique RND. A smooth and reliable RND is estimated guaranteeing non-negative density values, an expected behavior for tails, and an area under the curve near one. Results for estimated RND from S\&P500, are depicted in Figure 5 (solid line), and reveal the effectiveness of proposed methods. Estimation procedures were also applied to VIX, with similar data problems (Figure 4) as found for S\&P500. The same effect of information compatibility and smoothness is reflected for RND estimation, which is depicted in Figure 6 (solid line).

Mean pricing functions are not difficult to estimate, however, if we consider only call (put) prices a greater variability can be observed for those functions. This affects RND left tail estimation when using only calls, and RND right tail when using only puts. Considering an estimation procedure involving only calls, we obtain less intuitive and robust results, as can be perceived in Figure 7. In this case, it was considered a local cubic polynomial with bound constraints only related to mean, first and second derivatives. Further arbitrage constraints, related to the link between calls and puts, are missing. Due to the lack of structure, which is induced by joint contribution of calls and puts, results are less stable, and can even contradict basic theoretical results. On the other hand, if we do not consider open-interest weights in the optimization problem, former results are improved without achieving the effectiveness associated with the full structured model, as shown in Figure 7. Estimations were performed to S\&P500, and same type of results are obtained for VIX.

Following the suggestions of an anonymous referee, we agree that accuracy measures are needed for the analysis of the results. Bootstrap methods might be used to approximate confidence intervals for nonparametric estimates.

The implementation of such methods can be delicate as bootstrap algorithms' efficiency may depend on the data generation process, estimator's form, and their statistical properties. Results on bootstrap methods can be found in Efron (1982), Wu (1986), Härdle (1990), and Hall (2013). Particularly, when applied to nonparametric estimation and confidence intervals, we can refer to Efron and Tibshirani (1986), Hall (1988), Härdle and Bowman (1988), Cao-Abad (1991), Härdle and Marron (1991) and DiCiccio and Efron (1996). Wu (1986) and Liu (1988) are relevant for the application of bootstrap methods in RND estimation, the first accounts for the possibility of unbalanced regression data, and second for non-i.i.d. models. 

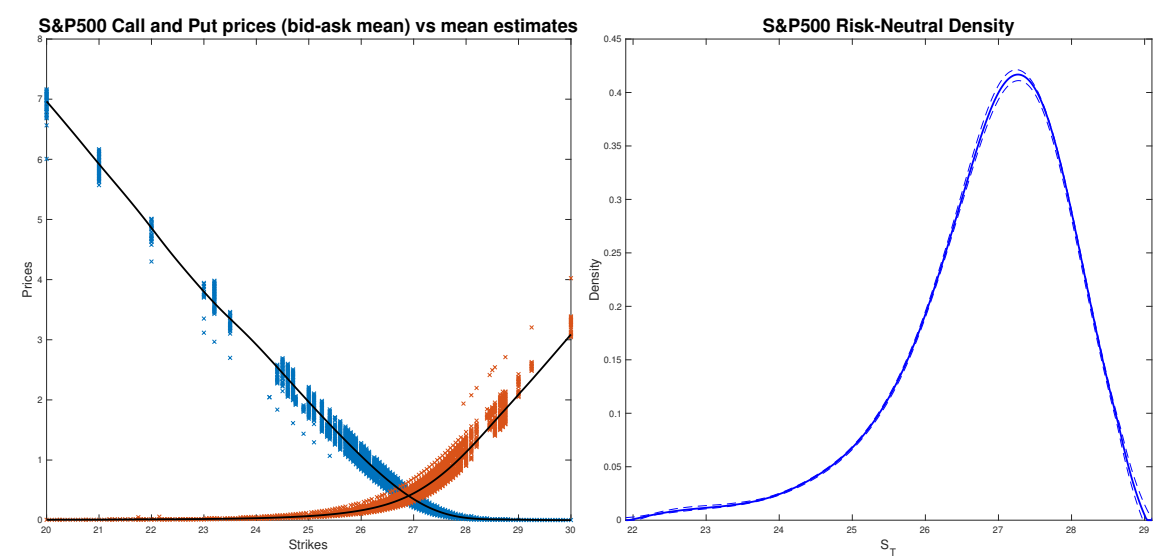

Fig. 5 Estimated call and put mean functions; RND estimates (solid line); and confidence intervals (dashed lines), for contracts with expiration date of May 18, $2018(\tau=1 / 12)$ on the S\&P500 index.
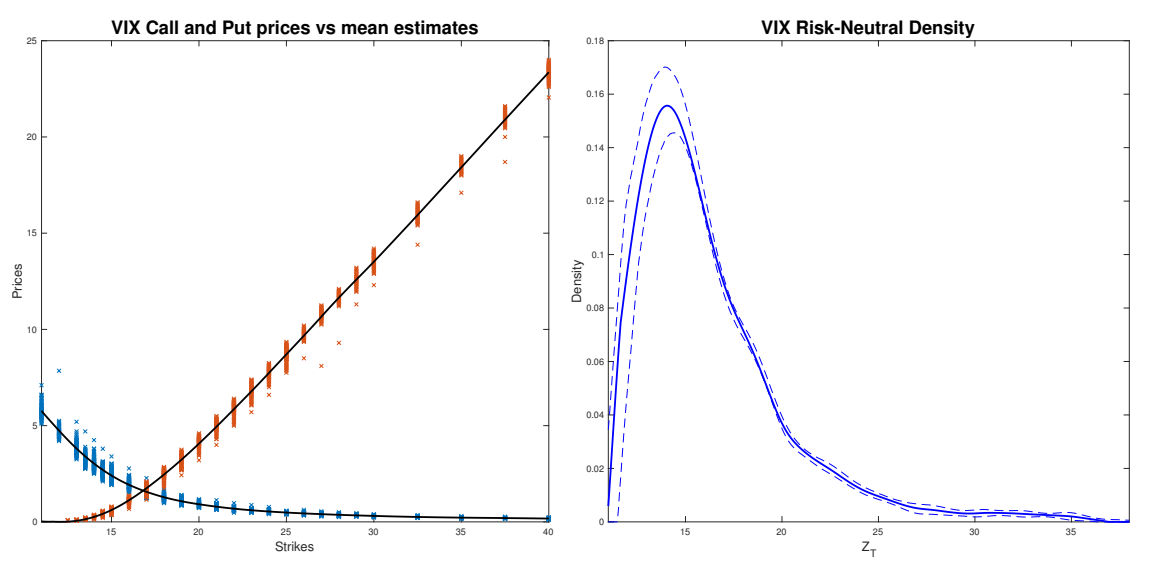

Fig. 6 Estimated call and put mean functions; RND estimates (solid line); and confidence intervals (dashed lines), for contracts with expiration date of May 16, $2018(\tau=1 / 12)$ on the VIX index.

Yatchew and Härdle (2006) used residuals and wild bootstrap. We use a pairs bootstrapping scheme, which is a more straightforward approach to implement. In fact, we softened our concerns with the well-known bias problem as samples of high dimension are considered. Also, we seek mainly to get the extent of confidence intervals amplitude, including directly the effect of heteroscedastic errors.

$\mathrm{Wu}$ (1986), in a linear regression context, using $X$ from the model $y=X \beta+e$, proposed to define a non-uniform bootstrapping resampling scheme. Also, Liu (1988) considered that "i.i.d. observations are drawn from an external population ... which is totally unrelated to the original data set". As standard pairs bootstrappring does not account for the possibility of non-i.i.d. data generating processes or unbalanced 

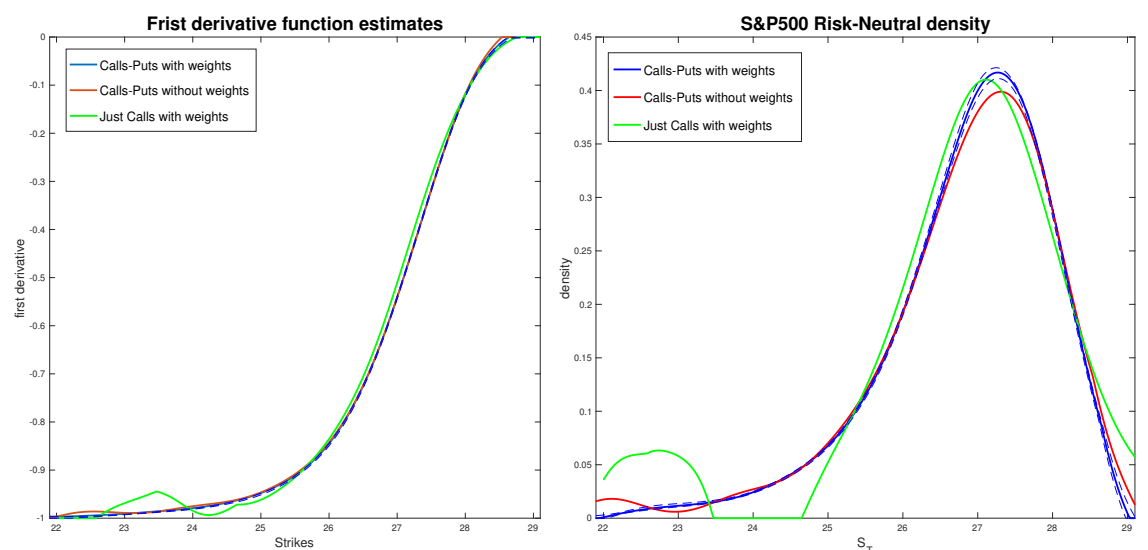

Fig. 7 Estimation results comparisons for S\&P500: only with calls, and without weights. Confidence intervals with dashed-lines.

samples, following both authors, we have also considered a non-uniform resampling scheme. In this case, bootstrapping probabilities are defined through the open-interest sample, which was also used to define weights in the criterion function associated with estimation. Open-interest data accounts for different amounts of information for each observation, mimicking the heteroscedasticity correction performed in a GLS setting. Without such new resampling scheme, bootstrap confidence intervals obtained are not interpretable.

Confidence intervals were approximated through 5000 iterations within the bootstrap method, and they are defined as the Monte Carlo sample quantiles. As depicted in Figures 5 and 6, we superimposed confidence intervals to estimated RNDs for S\&P500 and VIX, they give us an idea for the estimates' accuracy. As we can see, for S\&P500 confidence intervals are very narrow, for VIX's RND, they are wider near the mode, where the curvature takes more effect. VIX data is less symmetrical (Figure 4) around at-the-money region, however, still being compatible with accurate and interpretable estimates. These results are also comparable with ones presented in Song and Xiu (2016), whom for the same type of options found similar results in terms of confidence intervals, very narrow for S\&P500, and wider for VIX.

We mainly present confidence intervals for RND estimates, except for S\&P500 first derivative, Figure 7, which are barely discernible. Here, there is an overlap with Figure 5, to show that our method of including the contrast between calls and puts information and a weighting scheme based on open-interest values, can improve substantially RND estimation. Less efficient methods give estimates outside confidence intervals obtained from more robust methods. Confidence intervals were also calculated for mean functions, we do not present them here as they are incredible narrow, representing a high degree of accuracy. In fact, they are not very illustrative of the variability of estimates, in the same line of ones presented in Yatchew and Härdle (2006). 


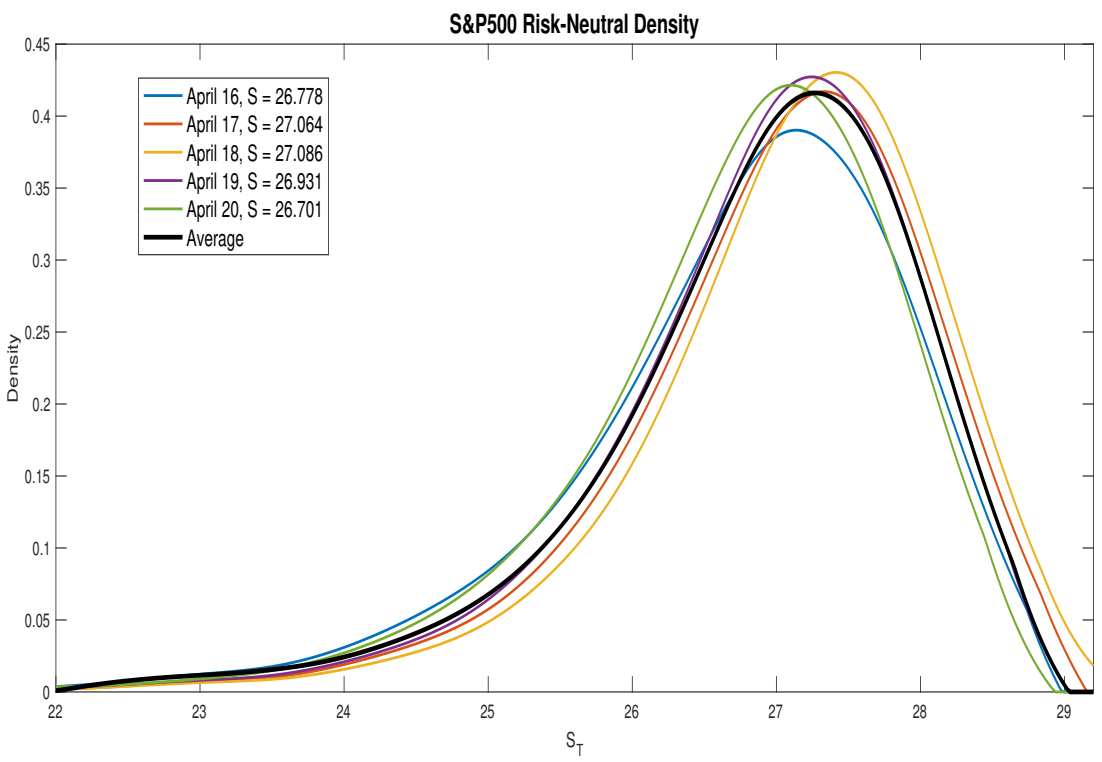

Fig. 8 One-day rolling window density estimation.

Having used one week of intraday data, obtained RNDs correspond to an average assuming that some factors influencing option prices are constant, apart from underlying asset price and volatility. To show the robustness of implemented methods, we compare the average density with densities obtained using one-day data in a rolling window. The results are depicted in Figure 8, where shapes of estimated densities do not vary significantly. Similar estimates are obtained, tails configurations are very robust, and the slight shifts in modes are essentially consequences of end-of-day price variation of the underlying asset. Results are presented for S\&P500 index options, considering VIX, similar results are obtained.

Finally, we have to highlight the difference in shape of both RND estimates (Figures 5 and 6). Results are intuitive and confirm what seems to be expected considering the different nature of the underlying assets. When subscribing S\&P500 options, the main interest is the left tail, since it reveals a possibility of drop in prices, or eventually a default. For VIX, the main interest is the right tail, as it is related to the possibility of an increase in volatility. This difference is clearly reflected in the estimation performed is this paper, and seems to reinforce the meaningfulness of proposed extensions.

\section{Concluding remarks}

This paper has developed and tested a new nonparametric approach for estimating RNDs from European option prices, using intraday data. The resulting problem is a quadratic programming problem, with a convex objective function, linear constraints, 
upper and lower bounds on variables. This is a challenging problem since RNDs are obtained through estimates for second derivatives. Naive approaches do not guarantee non-negativity, integration to one, and RND smoothness. Although, by defining a problem that includes calls, puts, and respective weights, it is guaranteed to obtain aforementioned features. Using simulated data we demonstrated that the method is able to recover, with acceptable accuracy, true RNDs. We applied the method to S\&P500 and VIX options with results that are robust and easily interpretable. Accuracy measures were also calculated through confidence intervals for estimates using bootstrap methods. Results confirm the robustness of proposed procedures. Comparison between both RNDs reveals main motivations for subscribing such securities: protection against decreases (S\&P500) and increases (VIX) on values of respective underlying assets.

Acknowledgements We are grateful to the Associate Editor and to an anonymous referee for many insightful comments and suggestions.

\section{References}

Aït-Sahalia, Y. and J. Duarte (2003). Nonparametric option pricing under shape restrictions. Journal of Econometrics 116(1), 9-47.

Aït-Sahalia, Y. and A. W. Lo (1998). Nonparametric estimation of state-price densities implicit in financial asset prices. The Journal of Finance 53(2), 499-547.

Aït-Sahalia, Y. and A. W. Lo (2000). Nonparametric risk management and implied risk aversion. Journal of Econometrics 94(1), 9-51.

Aït-Sahalia, Y., Y. Wang, and F. Yared (2001). Do option markets correctly price the probabilities of movement of the underlying asset? Journal of Econometrics 102(1), 67-110.

Banz, R. W. and M. H. Miller (1978). Prices for state-contingent claims: Some estimates and applications. Journal of Business, 653-672.

Birke, M. and K. F. Pilz (2008). Nonparametric option pricing with no-arbitrage constraints. Journal of Financial Econometrics 7(2), 53-76.

Breeden, D. T. and R. H. Litzenberger (1978). Prices of state-contingent claims implicit in option prices. Journal of Business, 621-651.

Cao-Abad, R. (1991). Rate of convergence for the wild bootstrap in nonparametric regression. The Annals of Statistics, 2226-2231.

Cox, J. C. and S. A. Ross (1976). The valuation of options for alternative stochastic processes. Journal of Financial Economics 3(1-2), 145-166.

Dalderop, J. (2018). Nonparametric filtering of conditional state-price densities. Technical report.

DiCiccio, T. J. and B. Efron (1996). Bootstrap confidence intervals. Statistical science, 189-212.

Efron, B. (1982). The jackknife, the bootstrap, and other resampling plans, Volume 38. Siam.

Efron, B. and R. Tibshirani (1986). Bootstrap methods for standard errors, confidence intervals, and other measures of statistical accuracy. Statistical science, 54-75. 
Fan, J. and I. Gijbels (1996). Local Polynomial Modelling and Its Applications, Volume 66. CRC Press.

Fengler, M. R. and L.-Y. Hin (2015). Semi-nonparametric estimation of the calloption price surface under strike and time-to-expiry no-arbitrage constraints. Journal of Econometrics 184(2), 242-261.

Grith, M., W. K. Härdle, and M. Schienle (2012). Nonparametric estimation of riskneutral densities. In Handbook of Computational Finance, pp. 277-305. Springer.

Hall, P. (1988). Theoretical comparison of bootstrap confidence intervals. The Annals of Statistics, 927-953.

Hall, P. (2013). The bootstrap and Edgeworth expansion. Springer Science \& Business Media.

Härdle, W. and A. W. Bowman (1988). Bootstrapping in nonparametric regression: Local adaptive smoothing and confidence bands. Journal of the American Statistical Association 83(401), 102-110.

Härdle, W. (1990). Applied Nonparametric Regression. Number 19. Cambridge university press.

Härdle, W. and J. Marron (1991). Bootstrap simultaneous error bars for nonparametric regression. The Annals of Statistics, 778-796.

Härdle, W. and Z. Hlávka (2009). Dynamics of state price densities. Journal of Econometrics 150(1), 1-15.

Heston, S. L. (1993). A closed-form solution for options with stochastic volatility with applications to bond and currency options. Review of Financial Studies 6(2), 327-343.

Jackwerth, J. C. (2000). Recovering risk aversion from option prices and realized returns. The Review of Financial Studies 13(2), 433-451.

Li, Q. and J. S. Racine (2007). Nonparametric Econometrics: Theory and Practice. Princeton University Press.

Liu, R. Y. (1988). Bootstrap procedures under some non-iid models. The Annals of Statistics 16(4), 1696-1708.

Monteiro, A. M., R. H. Tütüncü, and L. N. Vicente (2008). Recovering risk-neutral probability density functions from options prices using cubic splines and ensuring nonnegativity. European Journal of Operational Research 187(2), 525-542.

Nadaraya, E. A. (1964). On estimating regression. Theory of Probability \& Its Applications 9(1), 141-142.

Rosenberg, J. V. and R. F. Engle (2002). Empirical pricing kernels. Journal of Financial Economics 64(3), 341-372.

Song, Z. and D. Xiu (2016). A tale of two option markets: Pricing kernels and volatility risk. Journal of Econometrics 190(1), 176-196.

Watson, G. S. (1964). Smooth regression analysis. Sankhyā: The Indian Journal of Statistics, Series A, 359-372.

Wu, C.-F. J. (1986). Jackknife, bootstrap and other resampling methods in regression analysis. the Annals of Statistics 14(4), 1261-1295.

Yatchew, A. (2003). Semiparametric Regression for the Applied Econometrician. Cambridge University Press.

Yatchew, A. and W. Härdle (2006). Nonparametric state price density estimation using constrained least squares and the bootstrap. Journal of Econometrics 133(2), 
579-599.

Zhang, X., R. D. Brooks, and M. L. King (2009). A bayesian approach to bandwidth selection for multivariate kernel regression with an application to state-price density estimation. Journal of Econometrics 153(1), 21-32. 